\title{
Hopf Bifurcation and Stability Analysis of a Congestion Control Model with Delay in Wireless Access Network
}

\author{
Wen-bo Zhao, ${ }^{1}$ Xiao-ke Sun, ${ }^{2}$ and Huicheng Wang ${ }^{3}$ \\ ${ }^{1}$ School of Physics and Information, Tianshui Normal University, Tianshui, Gansu 741000, China \\ ${ }^{2}$ School of Mathematics and Statistics, Tianshui Normal University, Tianshui, Gansu 741000, China \\ ${ }^{3}$ Department of Mathematics, Zhejiang Normal University, Jinhua, Zhejiang 321004, China
}

Correspondence should be addressed to Huicheng Wang; yjs2014xia@163.com

Received 15 February 2014; Accepted 16 March 2014; Published 15 April 2014

Academic Editor: Yonghui Xia

Copyright (C) 2014 Wen-bo Zhao et al. This is an open access article distributed under the Creative Commons Attribution License, which permits unrestricted use, distribution, and reproduction in any medium, provided the original work is properly cited.

\begin{abstract}
We drive a scalar delay differential system to model the congestion of a wireless access network setting. The Hopf bifurcation of this system is investigated using the control and bifurcation theory; it is proved that there exists a critical value of delay for the stability. When the delay value passes through the critical value, the system loses its stability and a Hopf bifurcation occurs. Furthermore, the direction and stability of the bifurcating periodic solutions are derived by applying the normal form theory and the center manifold theorem. Finally, some examples and numerical simulations are presented to show the feasibility of the theoretical results.
\end{abstract}

\section{Introduction}

Recently, the wireless access network has been wildly applied to various fields, especially to the Internment; therefore, it has received significant attention. The congestion control in wireless access network also plays a crucial role in the success of the wireless network technology.

The congestion and avoidance mechanism is a combination of the end-to-end TCP congestion control mechanism [ 1 , 2 ] at the end hosts and the queue management mechanism at the routers. Because the congestion control algorithm is a highly complex dynamical model, many researchers have given much study to its dynamics and stability. In [3-5], the local stability in congestion control models is studied. In [69], the existence of Hopf bifurcation is analyzed in congestion control models.

For wired access network, the dynamic of window size is captured by the following equation [10]:

$$
\begin{array}{r}
\dot{W}_{i}(t)=x_{i}\left(t-\tau_{i}\right)\left(\frac{1-p_{i}(t)}{W_{i}(t)}-\frac{1}{2} p_{i}(t) W_{i}(t)\right), \\
i=1, \ldots, n,
\end{array}
$$

where $W_{i}(t), x_{i}(t)=W_{i}(t) / \tau_{i}, \tau_{i}$, and $p(t)$ denote the TCP window size, TCP rate, round trap time at time $t$ of flow $i$, and probability of packet mark at time $t$, respectively.

However, there are seldom works which discuss the dynamical behaviors of the congestion control model in wireless access network such as stability and Hopf bifurcation. The observation provides us with the motivation to investigate the dynamical behaviors of the congestion control model in wireless access network.

In this paper, we consider the wireless access networks of only one bottleneck router and let $n$ TCP flows tracer the router. In the down link communication from the network to the sources, the marking probability is fed back to the sources. During channel fading, the source has failed to receive the marking probability. Therefore, we suppose that the drop probability is $p_{d i}$. In this case, the source will use the previous packet marking probability to reduce its window size, and also the window size is decreased by one by convention. Thus, we obtain

$$
\begin{gathered}
\dot{W}_{i}(t)=x_{i}\left(t-\tau_{i}\right)\left(\frac{1-p_{i}(t)}{W_{i}(t)}-\frac{1}{2} p_{i}(t) W_{i}(t)\left(1-p_{d i}\right)\right. \\
\left.-p_{i}(t)\left(W_{i}(t)-1\right) p_{d i}\right) .
\end{gathered}
$$


The dynamic of queue length of the router is captured by the following equation [11]:

$$
\dot{q}(t)=F\left(\sum x_{i}(t-\tau)\right)-c,
$$

where $c$ is the serving capacity of the link node and the function $F\left(\sum x_{i}(t)\right)$ is the adjusted rate of the source based on the congestion rate $x(t)$ from the link node, which is a decreasing and nonnegative derivative function.

Since $x_{i}(t)=W_{i}(t) / \tau_{i}$ and $p_{i}(t)=k q(t)$ [12], we obtain

$$
\begin{aligned}
\dot{x}_{i}(t)=x_{i}\left(t-\tau_{i}\right)[ & \frac{1-k q(t)}{\tau_{i}^{2} x(t)}-\frac{1}{2} k x_{i}(t) q(t) \\
& \left.-\frac{1}{2} k p_{d i} x_{i}(t) q(t)+\frac{1}{\tau_{i}} k p_{d i} q(t)\right] .
\end{aligned}
$$

We assume that the $\tau_{i}$ is a constant and not timevarying and the queuing delay is neglected. So, we obtain the following congestion model in wireless network:

$$
\begin{aligned}
\dot{x}_{i}(t)=x_{i}(t-\tau)[ & \frac{1-k q(t)}{\tau^{2} x_{i}(t)}-\frac{1}{2} k x_{i}(t) q(t) \\
& \left.-\frac{1}{2} k p_{d} x_{i}(t) q(t)+\frac{1}{\tau} k p_{d} q(t)\right], \\
\dot{q}(t)= & F\left(\sum x_{i}(t-\tau)\right)-c .
\end{aligned}
$$

The paper is organized as follows. In Section 2, the stability of trivial solutions and the existence of Hopf bifurcation are discussed and the delay passes through the critical value, the system loses its stability, and a Hopf bifurcation occurs. In Section 3, based on the normal form theory and the center manifold theorem, we derive the formulas for determining the properties of the direction of the Hopf bifurcation and the stability of bifurcating periodic solutions. In Section 4, numerical simulations are given to justify the theoretical analysis. Finally, the conclusions appear in Section 5.

Since we focus on dynamical behavior analysis of the above model in the wireless access networks, we only need to choose the communication delay as the bifurcation parameter.

It is worth to point out that recent many works have been done for wired access network. For details, we refer to [13-16].

\section{Stability of the System with Communication Delay}

In this section, we assume that $x_{i}(t), i=1, \ldots, n$ is equal to $x(t)$, so (5) can be rewritten as follows:

$$
\begin{gathered}
\dot{x}(t)=x(t-\tau)\left[\frac{1-k q(t)}{\tau^{2} x(t)}-\frac{1}{2} k x(t) q(t)-\frac{1}{2} k p_{d} x(t) q(t)\right. \\
\left.+\frac{1}{\tau} k p_{d} q(t)\right], \\
\dot{q}(t)=F(n x(t-\tau))-c .
\end{gathered}
$$

Let the equilibrium point of the system (6) be $\left(x^{*}, q^{*}\right)$, which should satisfy

$$
\begin{gathered}
F\left(n x^{*}\right)=c \\
q^{*}=2\left[k\left(2+\left(1+p_{d}\right)\left(\tau x^{*}\right)^{2}-2 p_{d} \tau x^{*}\right)\right]^{-1}
\end{gathered}
$$

and $0<k q^{*} \leq 1$.

Hence, $\left(1+p_{d}\right)\left(\tau x^{*}\right)^{2}-2 p_{d} \tau x^{*}>0$ and we get

(H1) $\tau \geq 2 p_{d} / x^{*}\left(1+p_{d}\right)$.

Remark 1. Consider $\tau \in\left[\tau_{1},+\infty\right)$, where

$$
\tau_{1}=\frac{2 p_{d}}{x^{*}\left(1+p_{d}\right)} .
$$

Let $y_{1}(t)=x(t)-x^{*}, y_{2}(t)=q(t)-q^{*}$. Linearizing the system (6) about the equilibrium point, we get

$$
\begin{gathered}
\dot{y}_{1}(t)=a_{11} y_{1}(t)+a_{12} y_{1}(t-\tau)+b_{11} y_{2}(t), \\
\dot{y}_{2}(t)=a_{22} y_{1}(t-\tau),
\end{gathered}
$$

where

$$
\begin{gathered}
a_{11}=x^{*}\left[\frac{-1+k q^{*}}{\left(\tau x^{*}\right)^{2}}-\frac{1}{2} k q^{*}-\frac{1}{2} k p_{d} q^{*}\right] \\
a_{12}=\frac{1-k q^{*}}{\tau^{2} x^{*}}-\frac{1}{2} k x^{*} q^{*}+\frac{k p_{d} q^{*}}{\tau}-\frac{1}{2} k p_{d} x^{*} q^{*}, \\
b_{11}=x^{*}\left[-\frac{1}{2} k x^{*}-\frac{1}{2} k p_{d} x^{*}-\frac{k}{\tau^{2} x^{*}}+\frac{k p_{d}}{\tau}\right], \\
a_{22}=n D(F)\left(n x^{*}\right) .
\end{gathered}
$$

Then, the characteristic equation of the linearized equation (9) is

$$
D(\lambda, \tau)=\lambda^{2}-a_{11} \lambda-a_{12} \lambda e^{-\lambda \tau}-a_{22} b_{11} e^{-\lambda \tau}=0 .
$$

Note that the coefficients $a_{11}, a_{12}$, and $b_{11}$ depend on time delay $\tau$, since $q^{*}$ is connected with $\tau$. In order to apply the geometric criterion of Kuang $[17,18]$, we rewrite $D(\lambda, \tau)=0$ into

$$
D(\lambda, \tau)=P(\lambda, \tau)+Q(\lambda, \tau) e^{-\lambda \tau},
$$

where

$$
\begin{gathered}
P(\lambda, \tau)=\lambda^{2}-a_{11} \lambda, \\
Q(\lambda, \tau)=-a_{22} b_{11}-a_{12} \lambda .
\end{gathered}
$$

Lemma 2. If $(H 1)$ holds, then

(a) $P(0, \tau)+Q(0, \tau) \neq 0$;

(b) $P(\omega i, \tau)+Q(\omega i, \tau) \neq 0$ for all $\omega \in R$;

(c) $\lim \sup \{|Q(\lambda, \tau) / P(\lambda, \tau)|:|\lambda| \rightarrow+\infty, \operatorname{Re} \lambda \geq 0\}<1$;

(d) $F(\omega, \tau)=|P(\omega i, \tau)|^{2}-|Q(\omega i, \tau)|^{2}$ for each $\tau$ has at most a finite number of real zeros; 
(e) each positive root $\omega(\tau)$ of $F(\omega, \tau)=0$ is continuous and differentiable in $\tau$ whenever it exists.

Proof. (a) For $\tau \in\left[\tau_{1},+\infty\right)$,

$$
P(0, \tau)+Q(0, \tau)=-a_{22} b_{11} \neq 0 .
$$

(b) Consider $P(\omega i, \tau)+Q(\omega i, \tau)=-\omega^{2}-b_{11} a_{22}+i\left(-\omega a_{11}-\right.$ $\left.\omega a_{12}\right) \neq 0$.

(c) From (13), we get

$$
\lim _{|\lambda| \rightarrow+\infty}\left|\frac{Q(\lambda, \tau)}{P(\lambda, \tau)}\right|=\lim _{|\lambda| \rightarrow+\infty}\left|\frac{a_{12} \lambda-b_{11} a_{22}}{\lambda^{2}-a_{11} \lambda}\right|=0 .
$$

Hence, $\lim \sup \{|Q(\lambda, \tau) / P(\lambda, \tau)|:|\lambda| \rightarrow+\infty, \operatorname{Re} \lambda \geq 0\}=$ $0<1$.

(d) From (13), we get

$$
\begin{aligned}
F(\omega, \tau) & =|P(\omega i, \tau)|^{2}-|Q(\omega i, \tau)|^{2} \\
& =\omega^{4}+a_{11}^{2} \omega^{2}-a_{12}^{2} \omega^{2}-b_{11}^{2} a_{22}^{2} .
\end{aligned}
$$

Hence, (d) holds.

(e) $F(\omega, \tau)$ is continuous for $\omega$ and $\tau$ and differentiable in $\omega$; hence, implicit function theorem implies (e). This completes the proof of the theorem.

Supposing that $D(\omega i, \tau)=0$ and $\omega>0$, we get

$$
\begin{aligned}
& \sin \omega \tau=\frac{\omega\left(a_{11} a_{22} b_{11}-a_{12} \omega^{2}\right)}{a_{22}^{2} b_{11}^{2}+a_{12}^{2} \omega^{2}}, \\
& \cos \omega \tau=-\frac{\omega^{2}\left(a_{22} b_{11}-a_{11} a_{12}\right)}{a_{22}^{2} b_{11}^{2}+a_{12}^{2} \omega^{2}} .
\end{aligned}
$$

Hence,

$$
\begin{aligned}
F(\omega, \tau) & =|P(\omega i, \tau)|^{2}-|Q(\omega i, \tau)|^{2} \\
& =\omega^{4}+a_{11}^{2} \omega^{2}-a_{12}^{2} \omega^{2}-b_{11}^{2} a_{22}^{2} \\
& =0 .
\end{aligned}
$$

Let $z=\omega^{2}$, and then (18) can be rewritten as

$$
z^{2}+\left(a_{11}^{2}-a_{12}^{2}\right) z-b_{11}^{2} a_{22}^{2}=0 .
$$

Denote

$$
h(z, \tau)=z^{2}+\left(a_{11}^{2}-a_{12}^{2}\right) z-b_{11}^{2} a_{22}^{2} .
$$

Since $-b_{11}^{2} a_{22}^{2}<0$, the equation $h(z, \tau)=0$ has one positive root. We denote that the positive root is $z^{+}$. Then, (18) has positive real root $\omega\left(\sqrt{z^{+}}\right)$, where

$$
\omega=\omega(\tau)=\sqrt{\frac{-\left(a_{11}^{2}-a_{12}^{2}\right)+\sqrt{\left(a_{11}^{2}-a_{12}^{2}\right)^{2}+4 b_{11}^{2} a_{22}^{2}}}{2}} .
$$

For $\tau \in\left[\tau_{1},+\infty\right)$, let $\theta(\tau) \in(0,2 \pi)$ be defined by

$$
\begin{aligned}
& \sin \theta(\tau)=\frac{\omega\left(a_{11} a_{22} b_{11}-a_{12} \omega^{2}\right)}{a_{22}^{2} b_{11}^{2}+a_{12}^{2} \omega^{2}}, \\
& \cos \theta(\tau)=\frac{\omega^{2}\left(a_{22} b_{11}-a_{11} a_{12}\right)}{a_{22}^{2} b_{11}^{2}+a_{12}^{2} \omega^{2}},
\end{aligned}
$$

which combines with (18) and defines the following maps:

$$
S_{n}(\tau)=\tau-\frac{\theta(\tau)+2 n \pi}{\omega(\tau)}, \quad n \in N .
$$

According to [18] and the above discussion, we have the following result.

Theorem 3. Assume that (H1) is satisfied, and then $\lambda=$ $\pm \omega\left(\tau_{0}\right) i, \tau_{0} \in\left(0, \tau_{1}\right)$, are a pair of simple and conjugate pure imaginary roots of the characteristic equation (11) if and only if $S_{0}\left(\tau_{0}\right)=0$ for some $n \in N$. This pair of simple conjugate pure imaginary roots crosses the imaginary axis from left to right if $\delta\left(\tau_{0}\right)>0$ and crosses the imaginary axis from right to left if $\delta\left(\tau_{0}\right)<0$, where

$$
\delta\left(\tau_{0}\right):=\operatorname{sign}\left\{\left.\frac{d \operatorname{Re} \lambda}{d \tau}\right|_{\lambda=\omega\left(\tau_{0}\right) i}\right\}=\operatorname{sign}\left\{\left.\frac{d S_{n}(\tau)}{d \tau}\right|_{\tau=\tau_{0}}\right\} .
$$

By the the expression of $a_{11}, a_{12}$, and $b_{11}$, we know that they have singularity at $\tau=0$. We can not gain the conclusion that the equilibrium $\left(x^{*}, p^{*}\right)$ by discussing roots of the characteristic equation $D(\lambda, 0)=0$. To our knowledge, this case is rarely considered by papers. But we can get the stability of the system (6) when $\tau=\tau_{0} / 2$ by discussing the stability of the following auxiliary system:

$$
\begin{gathered}
\dot{y}_{1}(t)=c_{11} y_{1}(t)+c_{12} y_{1}(t-r)+d_{11} y_{2}(t), \\
\dot{y}_{2}(t)=c_{22} y_{1}(t-r),
\end{gathered}
$$

where

$$
\begin{aligned}
& c_{11}=\left.a_{11}\right|_{\tau=\tau_{0} / 2}, \\
& c_{12}=\left.a_{12}\right|_{\tau=\tau_{0} / 2}, \\
& c_{22}=\left.a_{22}\right|_{\tau=\tau_{0} / 2}, \\
& d_{11}=\left.b_{11}\right|_{\tau=\tau_{0} / 2},
\end{aligned}
$$

Then, the characteristic equation of the linearized equation (25) is

$$
\lambda-c_{11} \lambda-c_{12} \lambda e^{-\lambda r}-c_{22} d_{11} e^{-\lambda r}=0 .
$$

Definition 4. For simplicity, let

$$
D_{0}(\lambda, r)=\lambda^{2}-c_{11} \lambda-c_{12} \lambda e^{-\lambda r}-c_{22} d_{11} e^{-\lambda r} .
$$

Lemma 5. The equilibrium $(0,0)$ of system (25) is locally asymptotically stable when $r=0$. 
Proof. When $r=0$, (27) becomes

$$
\lambda^{2}-\left(c_{11}+c_{12}\right) \lambda-c_{22} d_{11}=0 .
$$

Further, if

(H2) $c_{11}+c_{12}<0$ and $c_{22} d_{11}<0$

is satisfied, all roots of (29) have negative real parts by the Routh-Hurwitz criteria. So, when $r=0$, the equilibrium point $(0,0)$ of system $(25)$ is locally asymptotically stable. This completes the proof of the lemma.

Let $\lambda= \pm i \omega_{r 0}$, where $\omega_{r 0}>0$. Substituting it into (27) and separating the real and imaginary parts, we have

$$
\begin{gathered}
-\omega_{r 0}^{2}-c_{12} \omega_{r 0} \sin \omega_{r 0} r-c_{22} d_{11} \cos \omega_{r 0} r=0, \\
-c_{11} \omega_{r 0}-c_{12} \omega_{r 0} \cos \omega_{r 0} r-c_{22} d_{11} \sin \omega_{r 0} r=0 .
\end{gathered}
$$

It follows from (30) that

$$
\begin{aligned}
& \sin \omega_{r 0} r=\frac{\omega_{r 0}\left(c_{11} c_{22} d_{11}-c_{12} \omega_{r 0}^{2}\right)}{c_{22}^{2} d_{22}^{2}+c_{12}^{2} \omega_{r 0}^{2}} \\
& \cos \omega_{r 0} r=-\frac{\omega_{r 0}^{2}\left(c_{22} d_{11}-c_{11} c_{12}\right)}{c_{22}^{2} d_{22}^{2}+c_{12}^{2} \omega_{r 0}^{2}}
\end{aligned}
$$

Since $\sin ^{2}\left(\omega_{r 0} r\right)+\cos ^{2}\left(\omega_{r 0} r\right)=1$, we have

$$
S_{0} \omega_{r 0}^{6}+S_{1} \omega_{r 0}^{4}+S_{2} \omega_{r 0}^{2}+S_{3}=0
$$

where

$$
\begin{gathered}
S_{0}=c_{12}^{2}, \\
S_{1}=c_{11}^{2} c_{12}^{2}+c_{22}^{2} d_{11}^{2}-c_{12}^{4}, \\
S_{2}=c_{11}^{2} c_{22}^{2} d_{11}^{2}-2 c_{12}^{2} c_{22}^{2} d_{11}^{2}, \\
S_{3}=-c_{22}^{4} d_{11}^{4} .
\end{gathered}
$$

Since $S_{0}>0$, we can rewrite (32) as

$$
\omega_{r 0}^{6}+R_{1} \omega_{r 0}^{4}+R_{2} \omega_{r 0}^{2}+R_{3}=0,
$$

where

$$
\begin{gathered}
R_{1}=\frac{c_{11}^{2} c_{12}^{2}+c_{22}^{2} d_{11}^{2}-c_{12}^{4}}{c_{12}^{2}}, \\
R_{2}=\frac{c_{11}^{2} c_{22}^{2} d_{11}^{2}-2 c_{12}^{2} c_{22}^{2} d_{11}^{2}}{c_{12}^{2}}, \\
R_{3}=\frac{-c_{22}^{4} d_{11}^{4}}{c_{12}^{2}} .
\end{gathered}
$$

Let $z=\omega_{r 0}^{2}$; then, (34) can be rewritten as

$$
z^{3}+R_{1} z^{2}+R_{2} z+R_{3}=0 .
$$

Denote

$$
h_{0}(z)=z^{3}+R_{1} z^{2}+R_{2} z+R_{3} .
$$

Since $\lim _{z \rightarrow+\infty} h_{0}(z)=+\infty$ and $R_{3}<0$, (36) has at the least one positive root. We define

$$
\Delta=\frac{4}{27} R_{2}^{3}-\frac{1}{27} R_{1}^{2} R_{2}^{2}+\frac{4}{27} R_{1}^{3} R_{3}-\frac{2}{3} R_{1} R_{2} R_{3}+R_{3}^{2} .
$$

Lemma 6. For cubic equation (36), the following cases need to be considered [19]:

(a) if $\Delta>0$, then the equation has three distinct real roots;

(b) if $\Delta=0$, then the equation has a multiple root and all its roots are real;

(c) if $\Delta<0$, then the equation has one real root and two nonreal complex conjugate roots.

Without loss of generality, we assume that (36) has three positive roots: $z_{01}, z_{02}$, and $z_{03}$. Since $z=\omega_{r 0}^{2}$ and $\omega_{r 0}>0$, we have

$$
\omega_{r 01}=\sqrt{z_{01}}, \quad \omega_{r 02}=\sqrt{z_{02}}, \quad \omega_{r 01}=\sqrt{z_{03}} .
$$

Thus, we know that

$$
\begin{array}{r}
r_{0 j}^{(s)}=\frac{1}{\omega_{r 0 j}}\left[\arccos \left(\frac{\omega_{r 0 j}^{2}\left(c_{22} d_{11}-c_{11} c_{12}\right)}{c_{22}^{2} b_{22}^{2}+c_{12}^{2} \omega_{r 0}^{2}}\right)+2 s \pi\right], \\
j=1,2,3 ; \quad s=0,1,2, \ldots .
\end{array}
$$

Denote

$$
r_{0}=\min _{j \in\{1,2,3\}}\left\{r_{0 j}^{(0)}\right\}
$$

Lemma 7. Assume that $\lambda= \pm i \omega_{r 0 j}$ are simple roots of (27) when $r=r_{0 j}^{(s)}$.

Proof. Since $D_{0}(\lambda, r)=\lambda^{2}-c_{11} \lambda-c_{12} \lambda e^{-\lambda r}-c_{22} d_{11} e^{-\lambda r}$, we obtain

$$
\frac{d D_{0}(\lambda)}{d \lambda}=2 \lambda-c_{11}+c_{22} d_{11} r e^{-\lambda r}-c_{12} e^{-\lambda r}+c_{12} \lambda r e^{-\lambda r}
$$

Substituting $\lambda=i \omega_{r}, r=r_{0}$ into (42), by using (30), we can obtain

$$
\begin{aligned}
\frac{d D_{0}\left(i \omega_{r}\right)}{d \lambda}= & -c_{11}+c_{22} d_{11} r_{0} \cos \left(\omega_{r 0} r_{0}\right)-c_{12} \cos \left(\omega_{r 0} r_{0}\right) \\
& +c_{12} \omega_{r 0} r_{0} \sin \left(\omega_{r 0} r_{0}\right) \\
& +i\left[2 \omega_{r 0}-c_{22} d_{11} r_{0} \sin \left(\omega_{r 0} r_{0}\right)-c_{12} \sin \left(\omega_{r 0} r_{0}\right)\right] \\
& +i\left[c_{12} \omega_{r 0} r_{0} \cos \left(\omega_{r 0} r_{0}\right)\right] \neq 0 .
\end{aligned}
$$

Similarly, we can get

$$
\frac{d D_{0}\left(-i \omega_{r}\right)}{d \lambda} \neq 0
$$

This completes the proof of the lemma. 
Hence, $\pm i \omega_{r 0 j}$ is a simple pair of purely imaginary roots of (27) with $r=r_{0 j}^{(s)}$.

Lemma 8. Let $\lambda(r)=\mu(r)+i \omega_{r}(r)$ be the root of (27) satisfying $\mu\left(r_{0}\right)=0, \omega_{r}\left(r_{0}\right)=\omega_{r 0}$; the following transversality condition holds:

$$
\left.\frac{d \operatorname{Re}(\lambda(r))}{d r}\right|_{r=r_{0}} \neq 0 .
$$

Proof. By equation (27) with respect to $r$ and applying the implicit function theorem, we get

$$
\frac{d \lambda(r)}{d r}=\frac{-\lambda e^{-\lambda r}\left(c_{22} d_{11}+c_{12} \lambda\right)}{2 \lambda-c_{11}+c_{22} d_{11} r e^{-\lambda r}-c_{12} e^{-\lambda r}+c_{12} \lambda r e^{-\lambda r}}
$$

Since $\lambda\left(r_{0}\right)=i \omega_{r 0}$, we obtain

$$
\operatorname{Re} \frac{d \lambda(r)}{d r}=\frac{p_{1}+p_{2} \sin \left(\omega_{r 0} r_{0}\right)+p_{3} \cos \left(\omega_{r 0} r_{0}\right)}{q_{1}+q_{2}}
$$

where

$$
\begin{gathered}
p_{1}=-c_{12}^{2} \omega_{r 0}, \quad p_{2}=-\omega_{r 0}\left(2 c_{12} \omega_{r 0}^{2}-c_{11} c_{22} d_{11}\right) \\
p_{2}=-\omega_{r 0}\left(c_{11} c_{12}-2 c_{22} d_{11}\right) \\
q_{1}=\left[-c_{11}+\left(c_{22} d_{11} r_{0}-c_{12}\right) \cos \left(\omega_{r 0} r_{0}\right)\right. \\
\left.+c_{12} \omega_{0} r_{0} \sin \left(\omega_{r 0} r_{0}\right)\right]^{2} \\
q_{2}=\left[2 \omega_{r 0}-\left(c_{22} d_{11} r_{0}-c_{12}\right) \sin \left(\omega_{r 0} r_{0}\right)\right. \\
\left.+c_{12} \omega_{0} r_{0} \cos \left(\omega_{r 0} r_{0}\right)\right]^{2}
\end{gathered}
$$

By again using (30), we can obtain

$$
\begin{gathered}
p_{1}+p_{2} \sin \left(\omega_{r 0} r_{0}\right)+p_{3} \cos \left(\omega_{r 0} r_{0}\right) \neq 0, \\
q_{1}+q_{2}>0 .
\end{gathered}
$$

Hence, $\left.(d \operatorname{Re}(\lambda(r)) / d r)\right|_{r=r_{0}} \neq 0$. This completes the proof of the lemma.

From the above discussion about the system (25), we have the following result.

Theorem 9. When $r<r_{0}$, the equilibrium point of system (25) is locally asymptotically stable.

Further, if

(H3) $r_{0}\left(\tau_{0}\right)>\tau_{0} / 2$

is satisfied, we will get the following lemma.

Lemma 10. The equilibrium point of system (6) is locally asymptotically stable when $\tau=\tau_{0} / 2$.

Proof. Since Lemma 5 and hypotheses (H3), the equilibrium point of system (25) is locally asymptotically stable when $r=$ $\tau_{0} / 2$. When $r=\tau_{0} / 2$ and $\tau=\tau_{0} / 2$, system (9) and system
(25) are the same system. So, there are no roots of $D\left(\lambda, \tau_{0} / 2\right)=$ $D_{0}\left(\lambda, \tau_{0} / 2\right)=0$ with nonnegative real parts and the equilibrium point of system (6) is locally asymptotically stable when $\tau=\tau_{0} / 2$. This completes the proof of the lemma.

According to [18] and the above discussion, we have following the result.

Theorem 11. Assume that (H1), (H2), and (H3) hold, if the function $S_{0}(\tau)$ has positive zeros in $\left(0, \tau_{1}\right)$; the equilibrium $\left(x^{*}, p^{*}\right)$ of system $(6)$ is asymptotically stable for all $\tau \in\left[\tau_{1}, \tau_{0}\right)$ and becomes unstable for staying in some right neighborhood of $\tau_{0}$; hence, system (6) undergoes Hopf bifurcation when $\tau=\tau_{0}$.

\section{Direction and Stability of the Hopf Bifurcation}

In this section, we will study the direction of Hopf bifurcation and the stability of bifurcating periodic solution of system (6) at $\tau=\tau_{0}$. The approach employed here is the normal form method and center manifold theorem introduced by Hassard [20]. More precisely, we will compute the reduced system on the center manifold with the pair of conjugate complex, purely imaginary solutions of the characteristic equation (11). By this reduction, we can determine the Hopf bifurcation direction, that is, to answer the question of whether the bifurcation branch of periodic solution exists locally for supercritical bifurcation or subcritical bifurcation.

Let $\tau=\tau_{0}+\mu, u_{i}(t)=y_{i}(\tau t),(i=1,2), \mu \in R, L_{\mu}: C \rightarrow$ $R^{2}$, and $F: R \times C \rightarrow R^{2}$, so that system (6) is transformed into an FDE in $C=C\left([-1,0], R^{2}\right)$ as

$$
\dot{u}(t)=L_{\mu}\left(u_{t}\right)+F\left(\mu, u_{t}\right)
$$

with

$$
\begin{gathered}
L_{\mu} \varphi=\left(\tau_{0}+\mu\right)[B \varphi(0)+C \varphi(-1)] \\
F_{1}(\varphi, \mu)=\left(\tau_{0}+\mu\right)\left[m_{1} \varphi_{1}^{2}(0)+m_{2} \varphi_{1}(0) \varphi_{1}(-1)\right. \\
+m_{3} \varphi_{1}(0) \varphi_{2}(0)+m_{4} \varphi_{1}(-1) \varphi_{2}(0) \\
+m_{5} \varphi_{1}^{3}(0)+m_{6} \varphi_{1}^{2}(0) \varphi_{1}(-1) \\
+m_{7} \varphi_{1}^{2}(0) \varphi_{2}(0) \\
\left.+m_{8} \varphi_{1}(0) \varphi_{1}(-1) \varphi_{2}(0)+\text { h.o.t }\right] \\
F_{2}(\varphi, \mu)=\left(\tau_{0}+\mu\right)\left[n_{1} \varphi_{1}^{2}(-1)+n_{2} \varphi_{1}^{3}(-1)\right]
\end{gathered}
$$

where

$$
\begin{gathered}
B=\left[\begin{array}{cc}
a_{11} & b_{11} \\
0 & 0
\end{array}\right], \quad C=\left[\begin{array}{ll}
a_{12} & 0 \\
a_{22} & 0
\end{array}\right], \\
m_{1}=\frac{1-k q^{*}}{\tau^{2}}, \\
m_{2}=\frac{-1+k q^{*}}{\left(\tau x^{*}\right)^{2}}-\frac{1}{2} k q^{*}-\frac{1}{2} k p_{d} q^{*},
\end{gathered}
$$




$$
\begin{gathered}
m_{3}=x^{*}\left(\frac{k}{\left(\tau x^{*}\right)^{2}}-\frac{1}{2} k-\frac{1}{2} k p_{d}\right), \\
m_{4}=-\frac{1}{2} k x^{*}-\frac{1}{2} k p_{d} x^{*}-\frac{k}{\tau^{2} x^{*}}+\frac{k p_{d}}{\tau}, \\
m_{5}=\frac{k q^{*}-1}{\tau^{2}\left(x^{*}\right)^{3}}, \\
m_{6}=\frac{1-k q^{*}}{\tau^{2}\left(x^{*}\right)^{3}}, \quad m_{7}=\frac{-k}{\left(x^{*} \tau\right)^{2}}, \\
m_{8}=\frac{k}{\left(x^{*} \tau\right)^{2}}-\frac{1}{2} k-\frac{1}{2} k p_{d}, \\
n_{1}=\frac{1}{2} n^{2} D^{(2)}(F)\left(n x^{*}\right), \\
n_{2}=\frac{1}{6} n^{3} D^{(3)}(F)\left(n x^{*}\right) .
\end{gathered}
$$

Then, $L_{\mu}$ is a one parameter family of bounded linear operator in $C\left([-1,0], \mathbb{R}^{2}\right)$. By the Riesz representation theorem, there exists a function $\eta(\theta, \mu)$ of bounded variation for $\theta \in[-1,0]$ such that

$$
L_{\mu} \phi=\int_{-1}^{0} d \eta(\theta, \mu) \phi(\theta), \quad \phi \in C
$$

In fact, we can choose

$$
\eta(\theta, \mu)=\left(\tau_{0}+\mu\right)[B \delta(\theta)-C \delta(\theta+1)],
$$

where $\delta(\theta)$ is Dirac delta function. For $\phi \in C^{1}\left([-1,0], R^{2}\right)$, the infinitesimal generator $A(\mu)$ is defined by

$$
A(\mu) \phi(\theta)= \begin{cases}\frac{d \phi}{d \theta}, & \theta \in[-1,0), \\ \int_{-1}^{0} d \eta(s, \mu) \phi(s), & \theta=0 .\end{cases}
$$

Further, let

$$
R(\mu) \phi(\theta)= \begin{cases}0, & \theta \in[-1,0), \\ F(\mu, \phi), & \theta=0,\end{cases}
$$

and then system (50) is equivalent to

$$
\dot{u}_{t}=A(\mu) u_{t}+R(\mu) u_{t}
$$

where $u_{t}(\theta)=u(t+\theta)$ for $\theta \in[-1,0]$.

The adjoint operator $A^{*}(\mu)$ of $A(\mu)$ is defined by

$$
A^{*}(\mu) \psi(\theta)= \begin{cases}-\frac{d \psi}{d \theta}, & \theta \in(0,1], \\ \int_{-1}^{0} \psi(-s) d \eta(s, \mu), & \theta=0,\end{cases}
$$

and a bilinear form

$$
\langle\psi, \phi\rangle=\bar{\psi}(0) \phi(0)-\int_{-1}^{0} \int_{0}^{\theta} \bar{\psi}(\xi-\theta) d \eta(\theta, \mu) \phi(\xi) d \xi,
$$

where $\psi \in C^{*}=C^{1}\left([0,1], R^{2 *}\right)$ and $R^{2 *}$ are row vector space.
Let $\mu=0$; by the discussion in the previous section, we know that $\pm \omega_{0} \tau_{0} i$ are common eigenvalues of $A(0)$ and $A^{*}(0)$. We need to compute the eigenvector of $A(0)$ and $A^{*}(0)$ corresponding to $\omega_{0} \tau_{0} i$ and $-\omega_{0} \tau_{0} i$, respectively. Suppose that $q(\theta)$ and $q^{*}(\theta)$ are the eigenvector of $A(0)$ and $A^{*}(0)$ corresponding to $\omega_{0} \tau_{0} i$ and $-\omega_{0} \tau_{0} i$, respectively; then, we have

$$
\begin{gathered}
A(0) q(\theta)=\omega_{0} \tau_{0} i q(\theta), \\
A^{*}(0) q^{*}(\theta)=-\omega_{0} \tau_{0} i q^{*}(\theta) .
\end{gathered}
$$

Then, we have the following lemma.

Lemma 12. Consider

$$
\begin{gathered}
q(\theta)=\left(\begin{array}{l}
1 \\
\gamma
\end{array}\right) e^{\omega_{0} \tau_{0} i \theta}, \quad \theta \in[-1,0], \\
q^{*}(s)=D\left(\begin{array}{ll}
1 & \left.\gamma^{*}\right) e^{\omega_{0} \tau_{0} i \theta}, \quad \theta \in[0,1], \\
\left\langle q^{*}, q\right\rangle=1, \quad\left\langle q^{*}, \bar{q}\right\rangle=0,
\end{array}\right.
\end{gathered}
$$

where

$$
\begin{gathered}
\gamma=\frac{a_{22} e^{-\omega_{0} \tau_{0} i}}{\omega_{0} i}, \quad \gamma^{*}=\frac{b_{11} i}{\omega_{0}}, \\
D=1+\gamma \overline{\gamma^{*}}-\tau_{0} a_{12} e^{-i \omega_{0} \tau_{0}}-\overline{\gamma^{*}} \tau_{0} a_{22} e^{-i \omega_{0} \tau_{0}} .
\end{gathered}
$$

Proof. From (55), we can rewrite (60) as

$$
\begin{gathered}
\frac{d q(\theta)}{d \theta}=i \omega_{0} \tau_{0} q(\theta), \quad \theta \in[-1,0), \\
\int_{-1}^{0} d \eta(s, 0) \varphi(s)=A(0) q(0)=i \omega_{0} \tau_{0} q(0), \quad \theta=0 .
\end{gathered}
$$

Based on (53) and (64), we have

$$
\tau_{0}[B q(0)+C q(-1)]=A(0) q(0)=i \omega_{0} \tau_{0} q(0) .
$$

For $q(-1)=q(0) e^{-i \omega_{0} \tau_{0}}$, we have

$$
\tau_{0}\left[B q(0)+C q(0) e^{-i \omega_{0} \tau_{0}}\right]=i \omega_{0} \tau_{0} q(0) .
$$

We can choose $q(0)=(1, \gamma)^{T}$ and get

$$
\begin{gathered}
\gamma=\frac{a_{22} e^{-\omega_{0} \tau_{0} i}}{\omega_{0} i}, \\
q(\theta)=(1, \gamma)^{T} e^{i \omega_{0} \tau_{0} \theta} .
\end{gathered}
$$

Similar to the proof of (64)-(68), we can obtain

$$
\begin{aligned}
q^{*}(0) & =\left(1, \frac{i b_{11}}{\omega_{0}}\right), \\
\gamma^{*} & =\frac{i b_{11}}{\omega_{0}} .
\end{aligned}
$$


Now, we can calculate $\left\langle q^{*}, q\right\rangle$ as

$$
\begin{aligned}
\left\langle q^{*}, q\right\rangle= & \bar{D}\left(1, \gamma^{*}\right)-\int_{-1}^{0} \int_{\xi=0}^{\theta} \bar{D}\left(1, \overline{\gamma^{*}}\right) e^{-i \omega_{0} \tau_{0}(\xi-\theta)}[d \eta(\theta)] \\
& \times(1, \gamma)^{T} e^{i \omega_{0} \tau_{0} \xi} d \xi \\
= & \bar{D}\left[1+\gamma \overline{\gamma^{*}}-\int_{-1}^{0}\left(1, \overline{\gamma^{*}}\right) \theta e^{i \omega_{0} \tau_{0} \theta}[d \eta(\theta)](1, \gamma)^{T}\right] \\
= & \bar{D}\left[1+\gamma \overline{\gamma^{*}}-\left(1, \overline{\gamma^{*}}\right)\left[-\tau_{0} C e^{-i \omega_{0} \tau_{0}}\right](1, \gamma)^{T}\right] \\
= & \bar{D}\left[1+\gamma \overline{\gamma^{*}}-\tau_{0} a_{12} e^{-i \omega_{0} \tau_{0}}-\overline{\gamma^{*}} \tau_{0} a_{22} e^{-i \omega_{0} \tau_{0}}\right]=1 .
\end{aligned}
$$

On the other hand, since $\langle\psi, A \varphi\rangle=\left\langle A^{*} \psi, \varphi\right\rangle$, we have

$$
\begin{aligned}
-i \omega_{0} \tau_{0}\left\langle q^{*}, \bar{q}\right\rangle & =\left\langle q^{*}, A \bar{q}\right\rangle \\
& =\left\langle A^{*} q^{*}, \bar{q}\right\rangle \\
& =\left\langle-i \omega_{0} \tau_{0} q^{*}, \bar{q}\right\rangle \\
& =i \omega_{0} \tau_{0}\left\langle q^{*}, \bar{q}\right\rangle
\end{aligned}
$$

Therefore, $\left\langle q^{*}, \bar{q}\right\rangle=0$. This completes the proof of the lemma.

In the remainder of this section, by using the same notations as in Hassard [20], we first compute the coordinates to describe the center manifold $C_{0}$ at $\mu=0$, which is a locally invariant, attracting two-dimensional manifold in $C_{0}$. Let $X_{t}$ be the solution of (57) when $\mu=0$. Define

$$
\begin{aligned}
z(t) & =\left\langle q^{*}, u_{t}\right\rangle, \\
W(t, \theta)=W(z, \bar{z}, \theta) & =u_{t}(\theta)-2 \operatorname{Re}\{z(t) q(\theta)\},
\end{aligned}
$$

and, then on the center manifold $C_{0}$, we have

$$
W(z, \bar{z}, \theta)=W_{20}(\theta) \frac{z^{2}}{2}+W_{11}(\theta) z \bar{z}+W_{02}(\theta) \frac{\bar{z}^{2}}{2}+\cdots
$$

and then $z$ and $\bar{z}$ are local coordinates for center manifold $C_{0}$ in the direction of $q$ and $q^{*}$. Note that $W$ is real if $u_{t}$ is real and we only deal with real solutions $u_{t}$. It is easy to see that

$$
\begin{aligned}
\dot{z}(t) & =\left\langle q^{*}, \dot{u}_{t}\right\rangle \\
& =\left\langle q^{*}, A(0) u_{t}+R(0) u_{t}\right\rangle \\
& =\left\langle q^{*}, A(0) u_{t}\right\rangle+\left\langle q^{*}, R(0) u_{t}\right\rangle \\
& =\left\langle A^{*}(0) q^{*}, u_{t}\right\rangle+\left\langle q^{*}, R(0) u_{t}\right\rangle
\end{aligned}
$$

$$
\begin{aligned}
& =i \omega_{0} \tau_{0} z(t)+\overline{q^{*}}(0) R(0) u_{t} \\
& \quad-\int_{-1}^{0} \int_{0}^{\theta} \overline{q^{*}}(\xi-\theta)[d \eta(\theta, 0)] R u_{t}(\xi) d \xi \\
& =i \omega_{0} \tau_{0} z(t)+\overline{q^{*}}(0) F\left(0, u_{t}(\theta)\right)-0 \\
& =i \omega_{0} \tau_{0} z(t)+\overline{q^{*}}(0) F_{0}(z(t), \bar{z}(t)) \\
& :=i \omega_{0} \tau_{0} z(t)+g(z, \bar{z}),
\end{aligned}
$$

where

$$
g(z, \bar{z})=g_{20} \frac{z^{2}}{2}+g_{11} z \bar{z}+g_{02} \frac{\bar{z}^{2}}{2}+g_{21} \frac{z^{2} \bar{z}}{2}+\cdots
$$

So, we can get

$$
\begin{aligned}
g(z, \bar{z}) & =\overline{q^{*}}(0) F_{0}(z(t), \bar{z}(t)) \\
& =\bar{D}\left(1, \overline{\gamma^{*}}\right)\left(F_{1}\left(0, u_{t}\right), F_{2}\left(0, u_{t}\right)\right)^{T},
\end{aligned}
$$

where

$$
\begin{aligned}
F_{1}\left(0, u_{t}\right)=\tau_{0}[ & m_{1} \varphi_{1}^{2}(0)+m_{2} \varphi_{1}(0) \varphi_{1}(-1) \\
& +m_{3} \varphi_{1}(0) \varphi_{2}(0)+m_{4} \varphi_{1}(-1) \varphi_{2}(0) \\
& +m_{5} \varphi_{1}^{3}(0)+m_{6} \varphi_{1}^{2}(0) \varphi_{1}(-1) \\
& +m_{7} \varphi_{1}^{2}(0) \varphi_{2}(0) \\
& \left.+m_{8} \varphi_{1}(0) \varphi_{1}(-1) \varphi_{2}(0)+\text { h.o.t }\right] \\
F_{2}\left(0, u_{t}\right) & =\tau_{0}\left[n_{1} \varphi_{1}^{2}(-1)+n_{2} \varphi_{1}^{3}(-1)\right]
\end{aligned}
$$

Since $u_{t}=u(t+\theta)=W(z, \bar{z}, \theta)+z q+\overline{z q}$ and $q(\theta)=$ $(1, \gamma) e^{i \omega_{0} \tau_{0} \theta}$, we have

$$
\begin{aligned}
u_{t}= & \left(\begin{array}{l}
u_{1}(t+\theta) \\
u_{2}(t+\theta)
\end{array}\right)=\left(\begin{array}{l}
W^{(1)}(t+\theta) \\
W^{(2)}(t+\theta)
\end{array}\right) \\
& +z\left(\begin{array}{l}
1 \\
\gamma
\end{array}\right) e^{i \omega_{0} \tau_{0} \theta}+\bar{z}\left(\frac{1}{\gamma}\right) e^{-i \omega_{0} \tau_{0} \theta}, \\
\varphi_{1}(0)= & z+\bar{z}+W_{20}^{(1)}(0) \frac{z^{2}}{2}+W_{11}^{(1)}(0) z \bar{z} \\
& +W_{02}^{(1)}(0) \frac{\bar{z}^{2}}{2}+\cdots, \\
\varphi_{2}(0)= & z \gamma+\overline{z \gamma}+W_{20}^{(2)}(0) \frac{z^{2}}{2}+W_{11}^{(2)}(0) z \bar{z} \\
& +W_{02}^{(2)}(0) \frac{\bar{z}^{2}}{2}+\cdots,
\end{aligned}
$$




$$
\begin{aligned}
\varphi_{1}(-1)= & z e^{-i \omega_{0} \tau_{0}}+\bar{z} e^{i \omega_{0} \tau_{0}}+W_{20}^{(1)}(-1) \frac{z^{2}}{2} \\
& +W_{11}^{(1)}(-1) z \bar{z}+W_{02}^{(1)}(-1) \frac{\bar{z}^{2}}{2}+\cdots, \\
\varphi_{2}(-1)= & z \gamma e^{-i \omega_{0} \tau_{0}}+\bar{z} \bar{\gamma} e^{i \omega_{0} \tau_{0}}+W_{20}^{(2)}(-1) \frac{z^{2}}{2} \\
& +W_{11}^{(2)}(-1) z \bar{z}+W_{02}^{(2)}(-1) \frac{\bar{z}^{2}}{2}+\cdots
\end{aligned}
$$

From the definition of $F\left(0, u_{t}\right)$, we have

$$
F_{0}(z, \bar{z})=\left(\begin{array}{l}
K_{11} z^{2}+K_{12} z \bar{z}+K_{13} \bar{z}^{2}+K_{14} z^{2} \bar{z} \\
K_{21} z^{2}+K_{22} z \bar{z}+K_{23} \bar{z}^{2}+K_{24} z^{2} \bar{z}
\end{array}\right)+\cdots,
$$

where

$$
\begin{aligned}
& k_{11}=\tau_{0}\left(m_{1}+m_{2} e^{-i \omega_{0} \tau_{0}}+m_{3} \gamma+m_{4} \gamma e^{-i \omega_{0} \tau_{0}}\right), \\
& k_{12}=\tau_{0}\left(2 m_{1}+m_{2} e^{i \omega_{0} \tau_{0}}+m_{2} e^{-i \omega_{0} \tau_{0}}+m_{3} \gamma\right. \\
& \left.+m_{3} \bar{\gamma}+m_{4} \gamma e^{i \omega_{0} \tau_{0}}+m_{4} \bar{\gamma} e^{-i \omega_{0} \tau_{0}}\right), \\
& k_{13}=\tau_{0}\left(m_{1}+m_{2} e^{i \omega_{0} \tau_{0}}+m_{3} \bar{\gamma}+m_{4} \bar{\gamma} e^{i \omega_{0} \tau_{0}}\right) \text {, } \\
& k_{14}=\tau_{0}\left(m_{1}\left(W_{20}^{(1)}(0)+2 W_{11}^{(1)}(0)\right)\right. \\
& +m_{2}\left(\frac{1}{2} W_{20}^{(1)}(0) e^{i \omega_{0} \tau_{0}}+W_{11}^{(1)}(0) e^{-i \omega_{0} \tau_{0}}\right. \\
& \left.+W_{11}^{(1)}(1)+\frac{1}{2} W_{20}^{(1)}(1)\right) \\
& +m_{3}\left(\frac{1}{2} \bar{\gamma} W_{20}^{(1)}(0)+\gamma W_{11}^{(1)}(0)\right. \\
& \left.+W_{11}^{(2)}(0)+\frac{1}{2} W_{20}^{(2)}(0)\right) \\
& +m_{4}\left(\frac{1}{2} W_{20}^{(2)}(0) e^{i \omega_{0} \tau_{0}}+W_{11}^{(2)}(0) e^{-i \omega_{0} \tau_{0}}\right. \\
& \left.+\gamma W_{11}^{(1)}(1)+\frac{1}{2} \bar{\gamma} W_{20}^{(1)}(1)\right) \\
& +3 m_{5}+m_{6}\left(e^{i \omega_{0} \tau_{0}}+2 e^{-i \omega_{0} \tau_{0}}\right)+m_{7}(\bar{\gamma}+2 \gamma) \\
& \left.+m_{8}\left(\gamma e^{i \omega_{0} \tau_{0}}+\bar{\gamma} e^{-i \omega_{0} \tau_{0}}+\gamma e^{-i \omega_{0} \tau_{0}}\right)\right), \\
& k_{21}=\tau_{0} n_{1} e^{-2 i \omega_{0} \tau_{0}}, \\
& k_{22}=2 \tau_{0} n_{1}, \\
& k_{23}=\tau_{0} n_{1} e^{2 i \omega_{0} \tau_{0}}, \\
& k_{24}=\tau_{0}\left(n_{1}\left(W_{20}^{(1)}(1) e^{i \omega_{0} \tau_{0}}+2 W_{11}^{(1)}(1) e^{-i \omega_{0} \tau_{0}}\right)\right. \\
& \left.+3 n_{2} e^{-i \omega_{0} \tau_{0}}\right) \text {. }
\end{aligned}
$$

Since $\overline{q^{*}}(0)=\bar{D}\left(1, \overline{\gamma^{*}}\right)$, we have

$$
\begin{aligned}
g(z, \bar{z})= & \overline{q^{*}}(0) F_{0}(z, \bar{z}) \\
= & \bar{D}\left(1, \overline{\gamma^{*}}\right)\left(\begin{array}{l}
K_{11} z^{2}+K_{12} z \bar{z}+K_{13} \bar{z}^{2}+K_{14} z^{2} \bar{z} \\
K_{21} z^{2}+K_{22} z \bar{z}+K_{23} \bar{z}^{2}+K_{24} z^{2} \bar{z}
\end{array}\right)+\cdots \\
= & \bar{D}\left[\left(K_{11}+\overline{\gamma^{*}} K_{21}\right) z^{2}+\left(K_{12}+\overline{\gamma^{*}} K_{22}\right) z \bar{z}\right. \\
& \left.+\left(K_{13}+\overline{\gamma^{*}} K_{23}\right) \bar{z}^{2}+\left(K_{14}+\overline{\gamma^{*}} K_{24}\right) z^{2} \bar{z}\right]
\end{aligned}
$$$$
+\cdots
$$

Comparing the coefficients of the above equation with those in (75), we have

$$
\begin{aligned}
& g_{20}=2 \bar{D}\left(K_{11}+\overline{\gamma^{*}} K_{21}\right), \\
& g_{11}=\bar{D}\left(K_{12}+\overline{\gamma^{*}} K_{22}\right), \\
& g_{02}=2 \bar{D}\left(K_{13}+\overline{\gamma^{*}} K_{23}\right), \\
& g_{21}=2 \bar{D}\left(K_{14}+\overline{\gamma^{*}} K_{24}\right) .
\end{aligned}
$$

In order to get the expression of $g_{21}$, we need to compute $W_{20}(\theta)$ and $W_{11}(\theta)$. Now, we determine the coefficients $W_{i j}(\theta)$ in (73). By (72) and (57), we have

$$
\begin{aligned}
& \dot{W}= \begin{cases}A(0) W-2 \operatorname{Re}\left\{\overline{q^{*}}(0) F_{0} q(\theta)\right\}, & -1 \leq \theta<0, \\
A(0) W-2 \operatorname{Re}\left\{\overline{q^{*}}(0) F_{0} q(\theta)\right\}+F_{0}, & \theta=0\end{cases} \\
& :=A(0) W+H(z, \bar{z}, \theta), \\
& H(z, \overline{z, \theta})=H_{20}(\theta) \frac{z^{2}}{2}+H_{11}(\theta) z \bar{z}+H_{02}(\theta) \frac{\bar{z}^{2}}{2}+\cdots
\end{aligned}
$$

From (73), (74), (83), and (84), we obtain

$$
\begin{gathered}
\left(2 i \omega_{0} \tau_{0}-A(0)\right) W_{20}(\theta)=H_{20}(\theta), \\
A(0) W_{11}(\theta)=-H_{11}(\theta) \\
\left(A(0)+2 i \omega_{0} \tau_{0}\right) W_{11}(\theta)=-H_{02}(\theta), \ldots .
\end{gathered}
$$

From (75) and (83), for $\theta \in[-1,0)$, we have

$$
\begin{aligned}
H(z, \bar{z}, \theta)= & -2 \operatorname{Re}\left\{\overline{q^{*}}(0) F_{0} q(\theta)\right\} \\
= & -2 \operatorname{Re}\{g(z, \bar{z}) q(\theta)\} \\
= & -g(z, \bar{z}) q(\theta)-\bar{g}(z, \bar{z}) \bar{q}(\theta) \\
= & -\left(g_{20} \frac{z^{2}}{2}+g_{11} z \bar{z}+g_{02} \frac{\bar{z}^{2}}{2}+g_{21} \frac{z^{2} \bar{z}}{2}\right) q(\theta) \\
& -\left(\bar{g}_{20} \frac{\bar{z}^{2}}{2}+\bar{g}_{11} z \bar{z}+\bar{g}_{02} \frac{z^{2}}{2}+\bar{g}_{21} \frac{\bar{z}^{2} z}{2}\right) \bar{q}(\theta) \cdots
\end{aligned}
$$


Comparing the coefficients of the above equation with those in (84), it follows that

$$
\begin{array}{r}
H_{20}(\theta)=-g_{20} q(\theta)-\bar{g}_{02} \bar{q}(\theta), \\
H_{11}(\theta)=-g_{11} q(\theta)-\bar{g}_{11} \bar{q}(\theta), \\
\theta \in[-1,0) .
\end{array}
$$

When $\theta=0$, we have

$$
\begin{aligned}
H(z, \bar{z}, 0)= & -2 \operatorname{Re}\left\{\overline{q^{*}}(0) F_{0} q(\theta)\right\}+F_{0} \\
= & -\left(g_{20} \frac{z^{2}}{2}+g_{11} z \bar{z}+g_{02} \frac{\bar{z}^{2}}{2}+g_{21} \frac{z^{2} \bar{z}}{2}\right) q(0) \\
& -\left(\bar{g}_{20} \frac{\bar{z}^{2}}{2}+\bar{g}_{11} z \bar{z}+\bar{g}_{02} \frac{z^{2}}{2}+\bar{g}_{21} \frac{\bar{z}^{2} z}{2}\right) \bar{q}(0) \\
& +F_{0} \\
= & -\left(g_{20} \frac{z^{2}}{2}+g_{11} z \bar{z}+g_{02} \frac{\bar{z}^{2}}{2}+g_{21} \frac{z^{2} \bar{z}}{2}\right) q(0) \\
& -\left(\bar{g}_{20} \frac{\bar{z}^{2}}{2}+\bar{g}_{11} z \bar{z}+\bar{g}_{02} \frac{z^{2}}{2}+\bar{g}_{21} \frac{\bar{z}^{2} z}{2}\right) \bar{q}(0) \\
& +\left(\begin{array}{l}
K_{11} z^{2}+K_{12} z \bar{z}+K_{13} \bar{z}^{2}+K_{14} z^{2} \bar{z} \\
K_{21} z^{2}+K_{22} z \bar{z}+K_{23} \bar{z}^{2}+K_{24} z^{2} \bar{z}
\end{array}\right)+\cdots
\end{aligned}
$$

Comparing the coefficients of the above equation with those in (84) gives that

$$
\begin{gathered}
H_{20}(0)=-g_{20} q(0)-\bar{g}_{02} \bar{q}(0)+2\left(K_{11}, K_{21}\right), \\
H_{11}(0)=-g_{11} q(0)-\bar{g}_{11} \bar{q}(0)+\left(K_{12}, K_{22}\right) .
\end{gathered}
$$

From (85) and the definition of $A(0)$, we have

$$
\begin{aligned}
\dot{W}_{20}(\theta) & =2 i \omega_{0} \tau_{0} W_{20}(\theta)+g_{20} q(\theta)+\bar{g}_{02} \bar{q}(\theta) \\
& =2 i \omega_{0} \tau_{0} W_{20}(\theta)+g_{20} q(0) e^{i \omega_{0} \tau_{0} \theta}+\bar{g}_{02} \bar{q}(0) e^{-i \omega_{0} \tau_{0} \theta} .
\end{aligned}
$$

Hence,

$$
W_{20}(\theta)=\frac{i g_{20} q(0)}{\omega_{0} \tau_{0}} e^{i \omega_{0} \tau_{0} \theta}+\frac{i \bar{g}_{02} \bar{q}(0)}{3 \omega_{0} \tau_{0}} e^{-i \omega_{0} \tau_{0} \theta}+E_{1} e^{2 i \omega_{0} \tau_{0} \theta}
$$

and, by a similar method, we get

$$
W_{11}(\theta)=-\frac{i g_{11} q(0)}{\omega_{0} \tau_{0}} e^{i \omega_{0} \tau_{0} \theta}+\frac{i \bar{g}_{11} \bar{q}(0)}{\omega_{0} \tau_{0}} e^{-i \omega_{0} \tau_{0} \theta}+E_{2},
$$

where $E_{1}$ and $E_{2}$ are both two-dimensional vectors. In the following, we will find out $E_{1}$ and $E_{2}$. From the definition of $A(0)$ and (85), we can obtain

$$
\int_{-1}^{0} d \eta(0, \theta) W_{20}(\theta)=2 i \omega_{0} \tau_{0} W_{20}(0)-H_{20}(0) .
$$

Notice that

$$
\begin{aligned}
&\left(i \omega_{0} \tau_{0} I-\int_{-1}^{0} d \eta(0, \theta) e^{i \omega_{0} \tau_{0} \theta}\right) q(0)= i \omega_{0} \tau_{0} q(0) \\
&-A(0) q(0)=0, \\
&\left(-i \omega_{0} \tau_{0} I-\int_{-1}^{0} d \eta(0, \theta) e^{-i \omega_{0} \tau_{0} \theta}\right) \bar{q}(0)=0 .
\end{aligned}
$$

Hence, we can obtain

$$
\left(2 i \omega_{0} \tau_{0} I-\int_{-1}^{0} d \eta(0, \theta) e^{2 i \omega_{0} \tau_{0} \theta}\right) E_{1}=2\left(\begin{array}{l}
K_{11} \\
K_{21}
\end{array}\right) .
$$

Similarly, we have

$$
\int_{-1}^{0} d \eta(0, \theta) E_{2}=-\left(\begin{array}{l}
K_{12} \\
K_{22}
\end{array}\right)
$$

Thus, we can get

$$
\begin{gathered}
\tau_{0}\left[\begin{array}{cc}
2 i \omega_{0}-a_{11}-a_{12} e^{-2 i \omega_{0} \tau_{0}} & -b_{11} \\
-a_{22} e^{-2 i \omega_{0} \tau_{0}} & 2 i \omega_{0}
\end{array}\right]\left[\begin{array}{l}
E_{1}^{(1)} \\
E_{1}^{(2)}
\end{array}\right]=\left[\begin{array}{l}
K_{12} \\
K_{22}
\end{array}\right], \\
\tau_{0}\left[\begin{array}{cc}
a_{11}+a_{12} & -b_{11} \\
a_{22} & 0
\end{array}\right]\left[\begin{array}{l}
E_{2}^{(1)} \\
E_{2}^{(2)}
\end{array}\right]=\left[\begin{array}{l}
-K_{12} \\
-K_{22}
\end{array}\right] .
\end{gathered}
$$

From (97), we can obtain

$$
\begin{aligned}
E_{1}^{(1)}= & -\left(b_{11} K_{21}+2 \omega_{0} K_{11}\right) \\
& \times\left(\tau _ { 0 } \left(-4 \omega_{0}^{2}+2 \omega_{0} a_{11}+2 \omega_{0} a_{11} e^{-2 i \omega_{0} \tau_{0}}\right.\right. \\
& \left.\left.+b_{11} a_{22} e^{-2 i \omega_{0} \tau_{0}}\right)\right)^{-1}, \\
E_{1}^{(2)}=- & \left(a_{22} K_{11} e^{-2 i \omega_{0} \tau_{0}}+2 K_{21} \omega_{0}-a_{11} K_{21}\right. \\
& \left.-a_{12} K_{21} e^{-2 i \omega_{0} \tau_{0}}\right) \\
\times & \left(\tau _ { 0 } \left(-4 \omega_{0}^{2}+2 \omega_{0} a_{11}+2 \omega_{0} a_{11} e^{-2 i \omega_{0} \tau_{0}}\right.\right. \\
& \left.\left.+b_{11} a_{22} e^{-2 i \omega_{0} \tau_{0}}\right)\right)^{-1}, \\
E_{2}^{(1)}=\frac{K_{22}}{a_{22} \tau_{0}} & \\
E_{2}^{(2)}= & \frac{a_{22} K_{12}-a_{11} K_{22}-a_{12} K_{22}}{a_{22} b_{11} \tau_{0}} .
\end{aligned}
$$

Based on the above analysis, we can determine $W_{20}(\theta)$ and $W_{11}(\theta)$ from (91) and (92). Furthermore, $g_{21}$ in (82) can be 


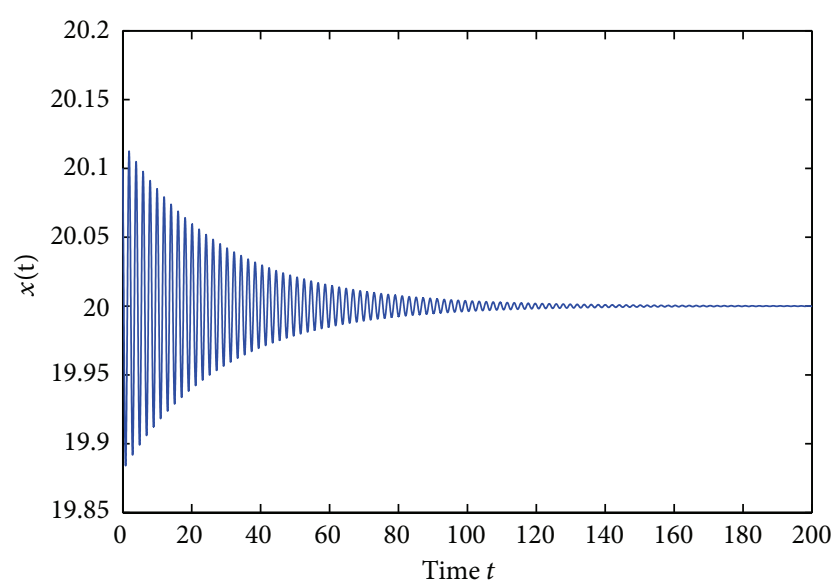

FIGURE 1: Waveform plot of $t-x(t)$ with $\tau=0.200$.

expressed by the parameters and delay. Thus, we can compute the following quantities:

$$
\begin{gathered}
c_{1}(0)=\frac{i}{2 \omega_{0} \tau_{0}}\left(g_{20} g_{11}-2\left|g_{11}\right|^{2}-\frac{\left|g_{02}\right|^{2}}{3}\right)+\frac{g_{21}}{2}, \\
\mu_{2}=-\frac{\operatorname{Re}\left\{c_{1}(0)\right\}}{\operatorname{Re}\left\{\lambda^{\prime}\left(\tau_{0}\right)\right\}}, \\
\beta_{2}=2 \operatorname{Re}\left\{c_{1}(0)\right\}, \\
T_{2}=-\frac{\operatorname{Im}\left\{c_{1}(0)\right\}+\mu_{2} \operatorname{Im}\left\{\lambda^{\prime}\left(\tau_{0}\right)\right\}}{\omega_{0} \tau_{0}},
\end{gathered}
$$

which determine the quantities of bifurcating periodic solutions in the center manifold at the critical value $\tau_{0}$ and we have the following result.

Theorem 13. In (99), the following results hold:

(i) the sign of $\mu_{2}$ determines the directions of the Hopf bifurcation: if $\mu_{2}>0(<0)$, then the Hopf bifurcation is supercritical (subcritical) and the bifurcating periodic solutions exist for $\tau>\tau_{0}\left(\tau<\tau_{0}\right)$;

(ii) the sign of $\beta_{2}$ determines the stability of the bifurcating periodic solutions: the bifurcating periodic solutions are stable (unstable) if $\beta_{2}<0\left(\beta_{2}>0\right)$;

(iii) the sign of $T_{2}$ determines the period of the bifurcating periodic solutions: the period increases (decreases) if $T_{2}>0\left(T_{2}<0\right)$.

\section{Numerical Simulation Examples}

In this section, we use the formulas obtained in Sections 2 and 3 to verify the existence of the Hopf bifurcation and calculate the Hopf bifurcation value and the direction of the Hopf bifurcation of system (6) with $c=1000, n=50$, and $k=0.001$.

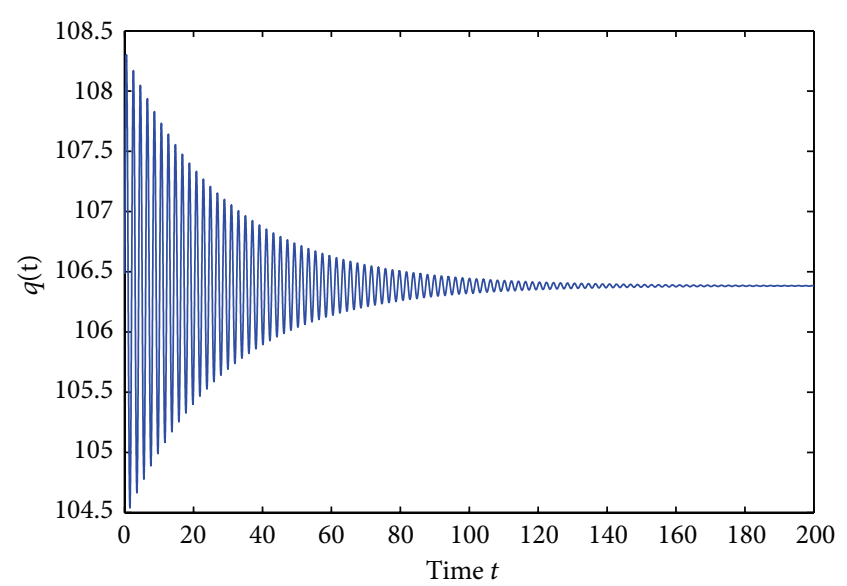

FIGURE 2: Waveform plot of $t-q(t)$ with $\tau=0.200$.

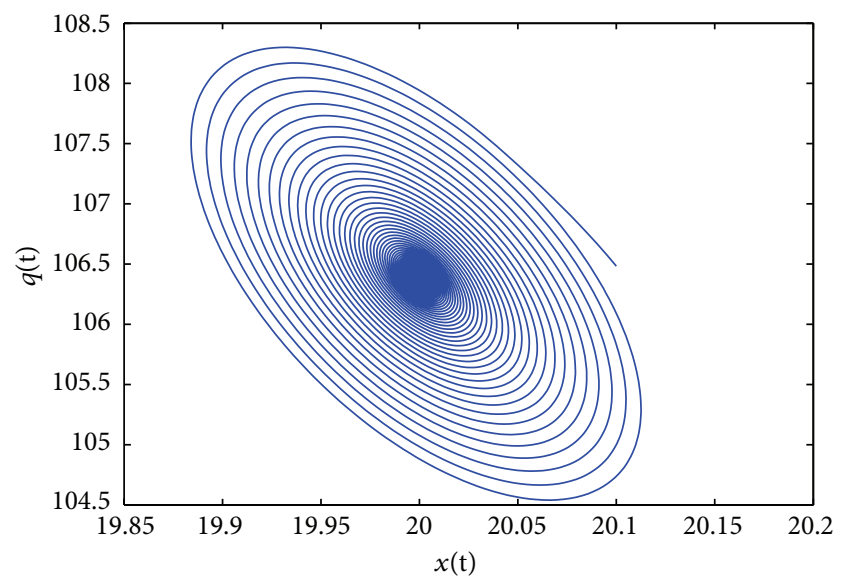

Figure 3: Phase plot for $x(t)-q(t)$ with $\tau=0.200$.

From (7), we have

$$
x^{*}=50, \quad q^{*}=\frac{1000}{1+220 \tau^{2}-2 \tau} .
$$

By calculation, we obtain that $\omega_{0} \approx 3.082, \tau_{0} \approx 0.203$, and $r_{0} \approx 0.472$. It follows from (3.32) that

$$
\begin{gathered}
c_{1}(0)=-0.000204+1.864768228 i, \\
\mu_{2}=0.000005120369502, \\
\beta_{2}=-0.4074400488 e-4, \\
T_{2}=-2.980526844 .
\end{gathered}
$$

These calculations prove that the system equilibrium $\left(x^{*}, q^{*}\right)$ is asymptotically stable when $\tau<\tau_{0}$ by computer simulation (see Figures 1, 2, and 3; $\tau=0.200$ ). When $\tau$ passes through the critical value $\tau_{0},\left(x^{*}, q^{*}\right)$ loses its stability and a Hopf bifurcation occurs (see Figures 4, 5, and 6; $\tau=0.206$ ).

\section{Conclusion}

A delayed model of congestion control was analyzed in this paper. Based on our theoretical analysis and numerical 


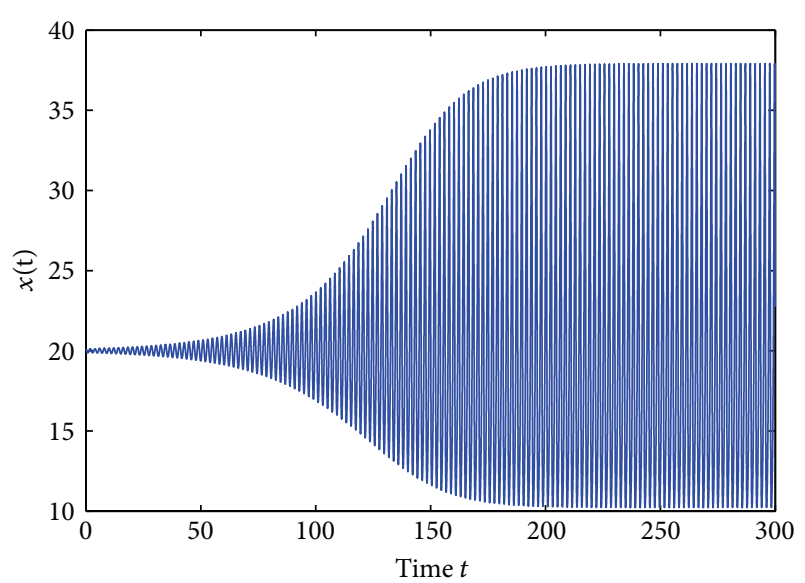

FIGURE 4: Waveform plot of $t-x(t)$ with $\tau=0.206$.

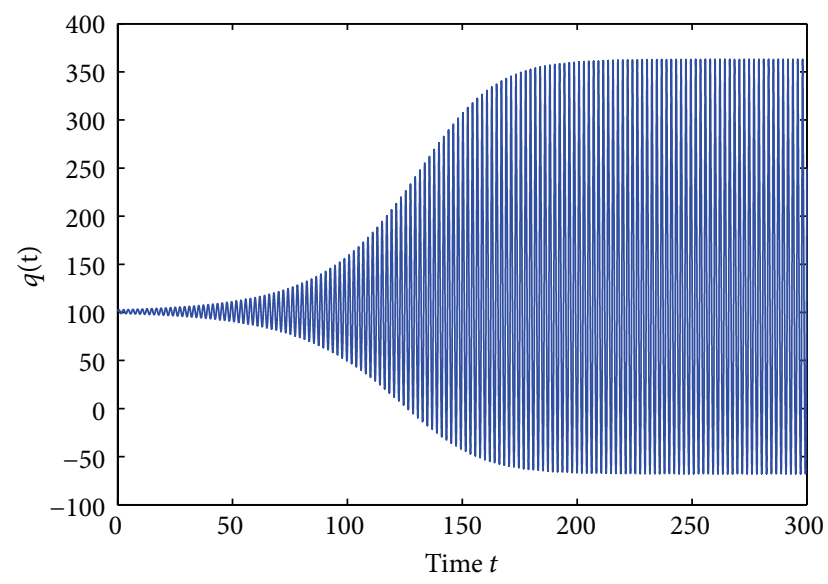

FIGURE 5: Waveform plot of $t-q(t)$ with $\tau=0.206$.

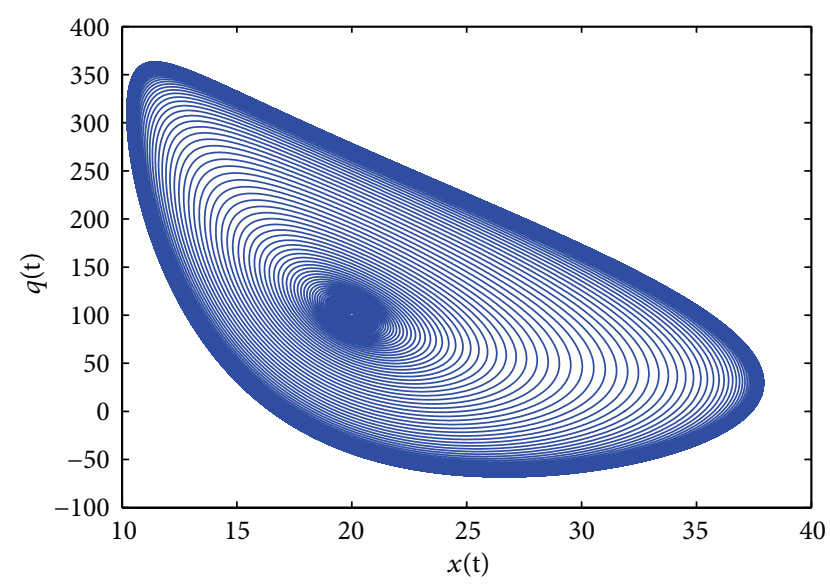

FIgURE 6: Phase plot for $x(t)-q(t)$ with $\tau=0.206$.

simulation, we can find that there exists a critical value for this delay and the whole system is stable when the delay of the system is less than this critical value. By using the time delay as a bifurcation parameter, we have shown that a Hopf bifurcation occurs when this parameter passes through a critical value, which means that the wireless access system will be congested, even collapsed, when the communication delay becomes large.

\section{Conflict of Interests}

The authors declare that there is no conflict of interests regarding the publication of this paper.

\section{References}

[1] S. H. Low, F. Paganini, J. Wang, and J. C. Doyle, "Linear stability of TCP/RED and a scalable control," Computer Networks, vol. 43, no. 5, pp. 633-647, 2003.

[2] V. Misra, W.-B. Gong, and D. Towsley, "Fluid-based analysis of a network of AQM routers supporting TCP flows with an application to RED," in Proceedings of the ACM SIGCOMM Computer Communication Review, pp. 151-160, September 2000.

[3] R. Yang, P. Shi, and G. P. Liu, "Filtering for discrete-time networked nonlinear systems with mixed random delays and packet dropouts," IEEE Transactions on Automatic Control, vol. 56, no. 11, pp. 2655-2660, 2011.

[4] H. C. Hsieh, J. S. Leu, and W. K. Shih, "Reaching consensus underlying an autonomous local wireless sensor network," International Journal of Innovative Computing, Information and Control, vol. 6, no. 4, pp. 1905-1914, 2010.

[5] N. Hamadneh, D. Murray, M. Dixon, and P. Cole, "Weighted RED (WTRED) strategy for TCP congestion control," in Informatics Engineering and Information Science, vol. 252 of Communications in Computer and Information Science, no. 2, pp. 421434, Springer, Berlin, Germany, 2011.

[6] F. S. Gentile, J. L. Moiola, and E. E. Paolini, "Nonlinear dynamics of internet congestion control: a frequency-domain approach," Communications in Nonlinear Science and Numerical Simulation, vol. 19, no. 4, pp. 1113-1127, 2014.

[7] S. Guo, H. Zheng, and Q. Liu, "Hopf bifurcation analysis for congestion control with heterogeneous delays," Nonlinear Analysis: Real World Applications, vol. 11, no. 4, pp. 3077-3090, 2010.

[8] D. Ding, J. Zhu, and X. Luo, "Hopf bifurcation analysis in a fluid flow model of Internet congestion control algorithm," Nonlinear Analysis: Real World Applications, vol. 10, no. 2, pp. 824-839, 2009.

[9] Y. G. Zheng and Z. H. Wang, "Stability and Hopf bifurcation of a class of TCP/AQM networks," Nonlinear Analysis: Real World Applications, vol. 11, no. 3, pp. 1552-1559, 2010.

[10] S. Guo, X. Liao, Q. Liu, and C. Li, "Necessary and sufficient conditions for Hopf bifurcation in exponential RED algorithm with communication delay," Nonlinear Analysis: Real World Applications, vol. 9, no. 4, pp. 1768-1793, 2008.

[11] H. Y. Yang and Y. P. Tian, "Hopf bifurcation in REM algorithm with communication delay," Chaos, Solitons and Fractals, vol. 25, no. 5, pp. 1093-1105, 2005.

[12] C. V. Hollot, V. Misra, D. Towsley, and W. Gong, "Analysis and design of controllers for AQM routers supporting TCP flows," IEEE Transactions on Automatic Control, vol. 47, no. 6, pp. 945959, 2002.

[13] D. W. Ding, X. M. Qin, N. Wang et al., "Hybrid control of Hopf bifurcation in a dual model of Internet congestion control system," Nonlinear Dynamics, pp. 1-10, 2013.

[14] F. S. Gentile, J. L. Moiola, and E. E. Paolini, "Nonlinear dynamics of internet congestion control: a frequency-domain approach," 
Communications in Nonlinear Science and Numerical Simulation, vol. 19, no. 4, pp. 1113-1127, 2014.

[15] F. Liu, G. Xiong, Z. H. Guan et al., "Stability analysis and control Hopf bifurcation in a FAST TCP model," in Proceedings of the IEEE 32nd Chinese Control Conference (CCC '13), pp. 1076-1080, 2013.

[16] M. Xiao, G. Jiang, and L. Zhao, "State feedback control at Hopf bifurcation in an exponential RED algorithm model," Nonlinear Dynamics, pp. 1-16, 2014.

[17] Y. Kuang, Delay Differential Equations: With Applications in Population Dynamics, Academic Press, New York, NY, USA, 1993.

[18] E. Beretta and Y. Kuang, "Geometric stability switch criteria in delay differential systems with delay dependent parameters," SIAM Journal on Mathematical Analysis, vol. 33, no. 5, pp. 11441165, 2002.

[19] R. S. Irving, Integers, Polynomials, and Rings: A Course in Algebra, Springer, Berlin, Germany, 2004.

[20] B. D. Hassard, Theory and Applications of Hopf Bifurcation, CUP Archive, 1981. 


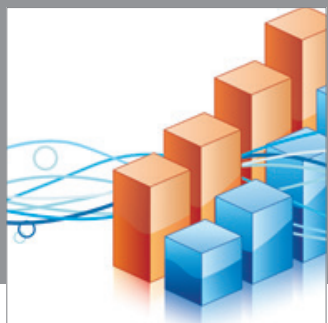

Advances in

Operations Research

mansans

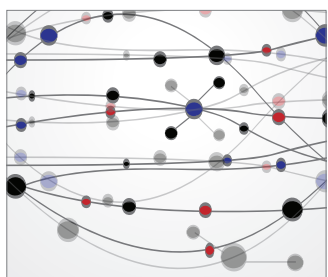

The Scientific World Journal
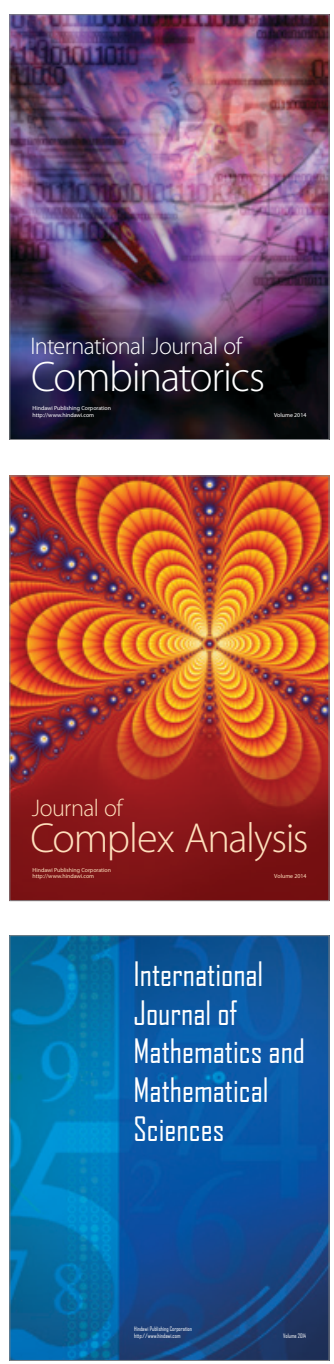
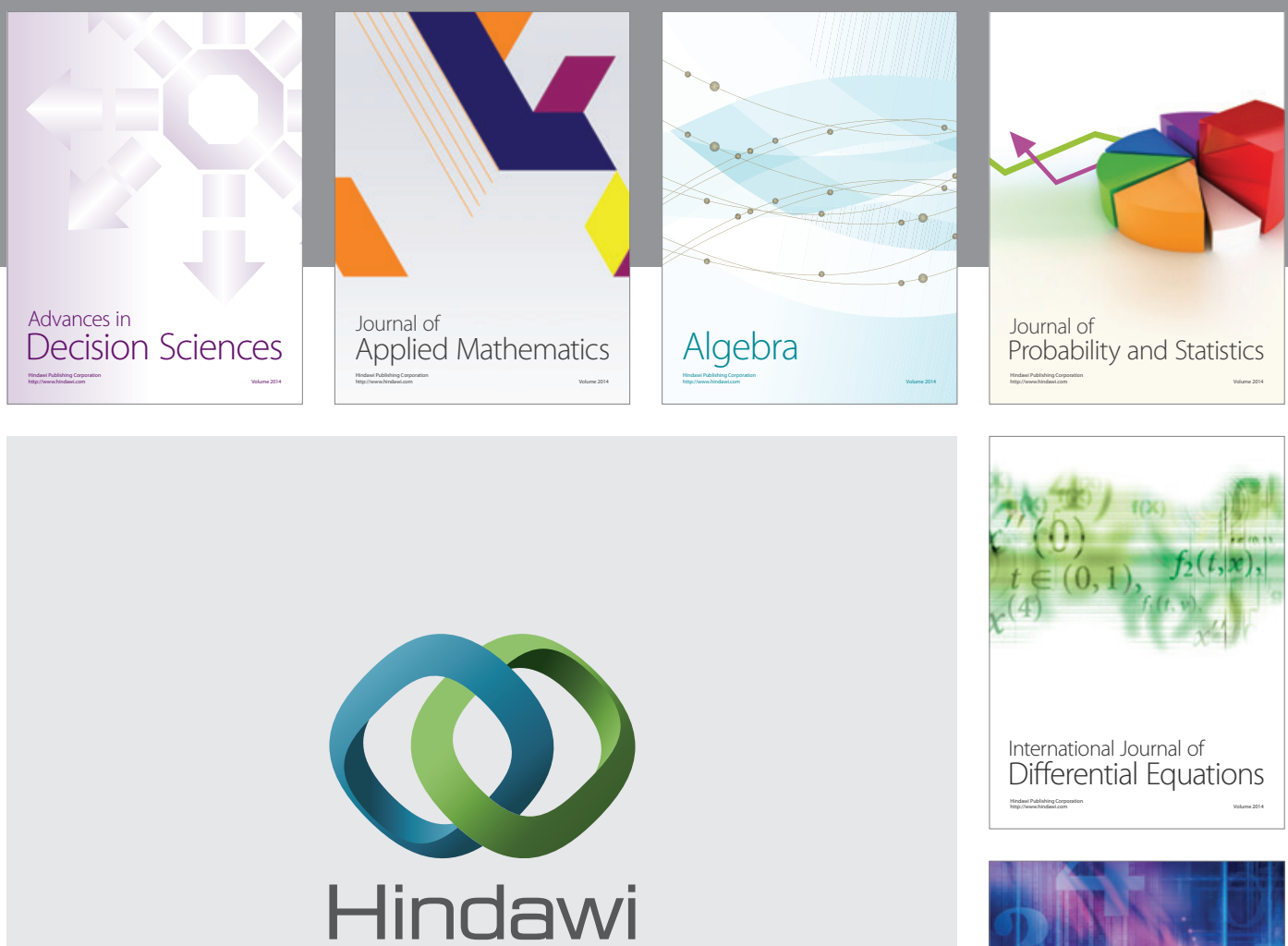

Submit your manuscripts at http://www.hindawi.com
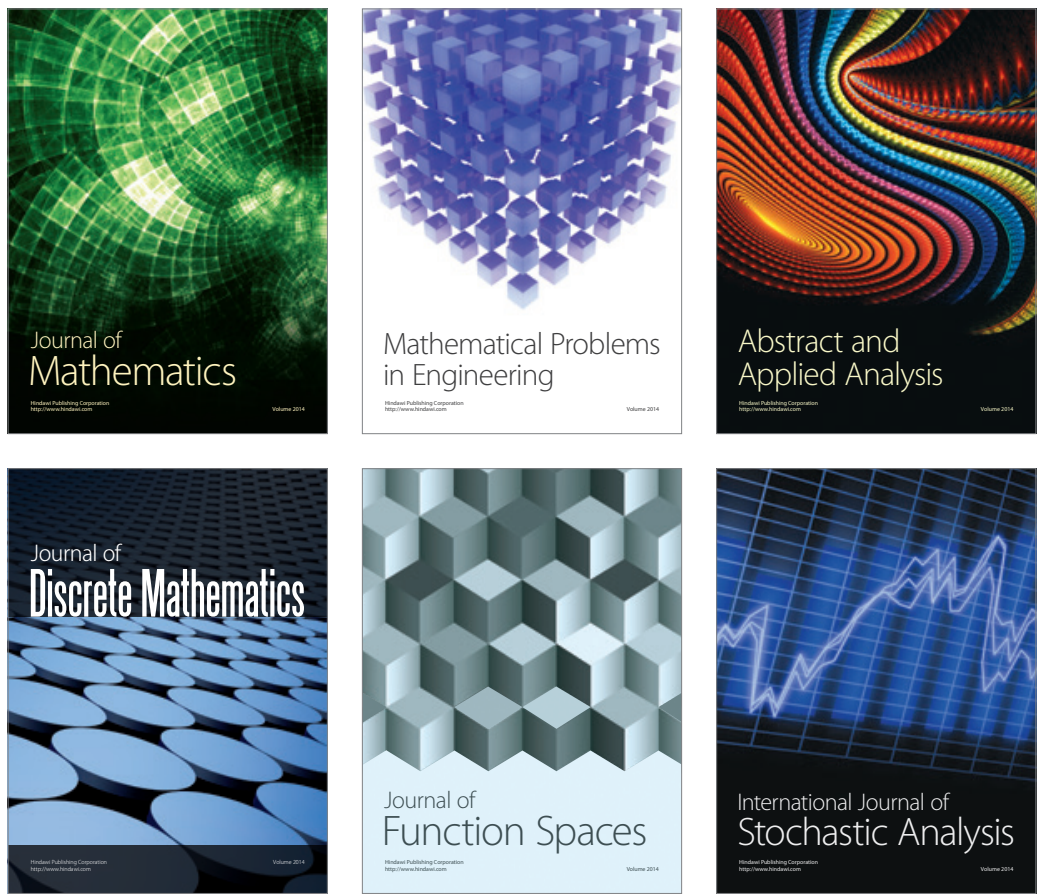

Journal of

Function Spaces

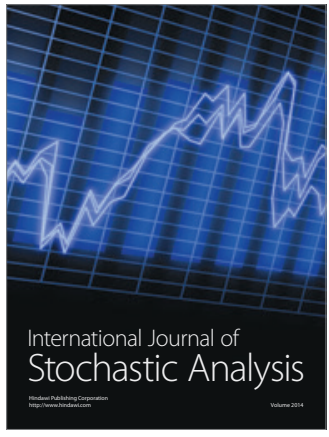

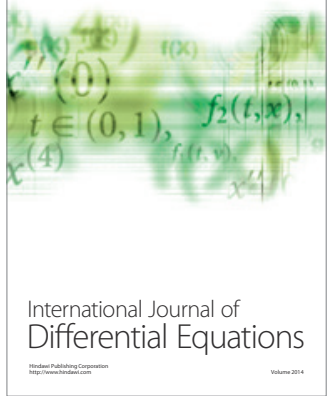
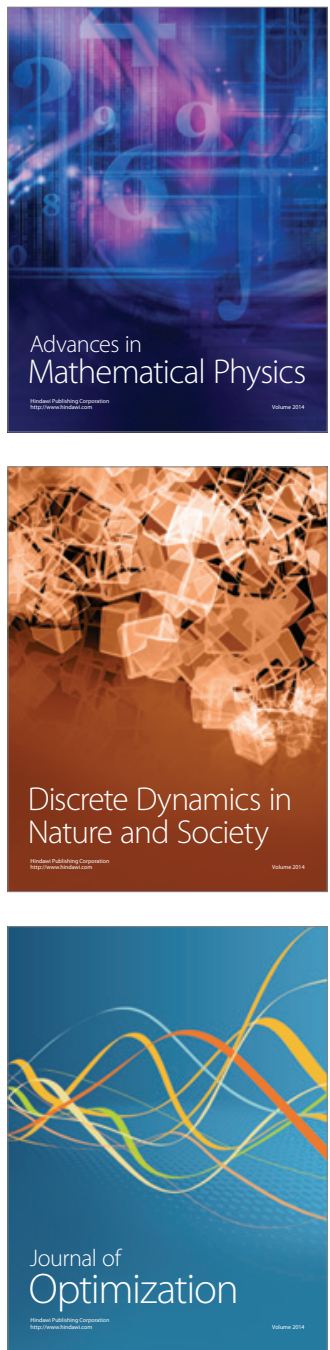